\title{
"Attenuated Psychosis Syndrome" or "Subthreshold Prodromal State"?
}

\author{
Vassilis Kontaxakis $^{1 凶}{ }^{\bowtie}$, Beata Havaki-Kontaxaki², Konstantinos Kollias², \\ Panagiotis Ferentinos ${ }^{1}$ and George Papadimitriou ${ }^{2}$
}

'Second Department of Psychiatry, Athens University Medical School, "Attikon" General Hospital, Athens, Greece ${ }^{2}$ First Department of Psychiatry, Athens University Medical School, "Eginition" Hospital, Athens, Greece

\author{
Dear editor, \\ Psychotic disorders, such as schizophrenia, are the most
} disabling mental illnesses affecting, usually, young people. Several projects have recently focused on people with prodromal symptoms but not frankly psychotic. There is evidence that interventions in early stages of the disease could result in better outcomes. ${ }^{1}$ In 1987, the DSM-III-R (American Psychiatric Association) provided a list of nine observable behavioral or subjectively experienced changes for the diagnosis of the "schizophrenia prodrome", but the list of criteria dropped from DSM-IV (1994). The ICD-10 (World Health Organization, 1994) acknowledges a prodrome as part of schizophrenia but this is not included in the description of the disorder because of its poor reliability and low sensitivity. ${ }^{2} \mathrm{~A}$ workgroup in the American Psychiatric Association has recently recommended inclusion of a new diagnostic category entitled "Attenuated Psychosis Syndrome" in the forthcoming DSM-5. However, the group is examining the evidence as to whether inclusion is merited in the main manual or in an appendix for further research.

It should be noted that subthreshold conditions, which are common in everyday practice, are characterized by symptoms likely below the threshold according to which categorical diagnosis is made; these conditions could progress or not to major psychiatric disorders. ${ }^{3.4}$ During the last decade, there was a vivid debate as to whether "Attenuated Psychosis Syndrome"

Received: May 12, 2012 Revised: November 2, 2012

Accepted: January 2, 2013 Available online: May 30, 2013

$\triangle$ Correspondence: Vassilis Kontaxakis, MD

Second Department of Psychiatry, Athens University Medical School, "Attikon" General Hospital, 1 Rimini St. 12462 Athens, Greece

Tel: +30-210-5832429, Fax: +30-210-5326453

E-mail: editor@psych.gr

(a) This is an Open Access article distributed under the terms of the Creative Commons Attribution Non-Commercial License (http://creativecommons.org/licenses/bync/3.0) which permits unrestricted non-commercial use, distribution, and reproduction in any medium, provided the original work is properly cited. should be included or not in DSM-5. ${ }^{5}$ Those in favor claim that subjects with the syndrome exhibit deficits at a psychological, behavioral and cognitive level (possibly subthreshold), have the right and need to be treated. The widely accepted "neurodevelopmental theory" of schizophrenia provides an implicit theoretical foundation for early intervention during the pre-psychotic stage of the illness. The identification of individuals carrying unexpressed genetic liability to psychosis will be crucial for both etiological research and clinical risk stratification. Moreover, according to the "clinical stage model", the earlier in the course of illness treatment is offered the greater remission and recovery rates are. ${ }^{1,6}$ Those against emphasize the risk of stigmatization of people with minor psychopathology, the fact that only a minority will convert to psychosis in the following years and that antipsychotic medication in non-psychotic populations may be unethical. However, antipsychotic medication has already been used with good results in non-psychotic conditions, such as personality disorders (schizotypal, borderline, etc.) or behavioral disturbances (impulsivity, aggression, etc.).

Stigmatization of people with the syndrome could be avoided if the word "psychosis" dropped from the title. Therefore, our proposal is that this condition could be included in DSM-5 as a new category for further research with the title "Subthreshold Prodromal State". "Subthreshold" because of the decreased severity of psychotic symptoms, "Prodromal" both because the term has been associated with psychosis and because the subjects could manifest major psychopathology in the future and "State" because diagnosis may change with time.

\section{REFERENCES}

1. McGorry PP, Killackey E, Yung A. Early intervention in psychosis: concepts, evidence and future directions. World Psychiatry 2008;7:148-156.

2. Simon AE, Ferrero FP, Merlo MC. Prodromes of first episode psychosis: how can we challenge nonspecifity? Compr Psychiatry 2001;42: 
382-392.

3. Shankman SA, Lewinsohn PM, Klein DN, Small JW, Seeley JR, Altman SE. Subthreshold conditions as precursors for full syndrome disorders: a 15-year longitudinal study of multiple diagnostic classes. J Child Psychol Psychiatry 2009;50:1485-1494.

4. Lewinsohn PM, Shankman SA, Gau JM, Klein DN. The prevalence and co-morbidity of subthreshold psychiatric conditions. Psychol Med 2004;34:613-622.
5. Shrivastava A. Should 'risk syndrome for psychosis' be included as a diagnosis in DSM-V? World Psychiatry 2010;9:123.

6. Klosterkötter J, Shultze-Lutter F, Bechdolf A, Ruhrmann S. Prediction and prevention of schizophrenia: what has been achieved and where to go next? World Psychiatry 2011;10:165-174.

7. Ravindran AV, Bradbury C, McKay M, da Silva TL. Novel uses for risperidone: focus on depressive, anxiety and behavioral disorders. Expert Opin Pharmacother 2007;8:1693-1710. 